\title{
ERRATUM
}

\section{Stability analysis of paraplegic standing while wearing an orthosis}

\author{
Takahiro Kagawa · Hiroshi Fukuda \\ Yoji Uno
}

Published online: 13 September 2006

(C) International Federation for Medical and Biological Engineering 2006

\section{Erratum to: Med Biol Eng Comput DOI 10.1007/s11517-006-0087-4}

Unfortunately, the order of the authors' names was incorrect.

The correct names should read Hiroshi Fukuda and Yoji Uno.

The online version of the original article can be found at http://dx.doi.org/10.1007/s11517-006-0087-4.

T. Kagawa $(\bowtie)$

Department of Biosciences and Informatics,

Faculty of Science and Technology, Keio University,

3-14-1 Hiyoshi, Kohokuku, Yokohama-shi 223-8522, Japan

e-mail: kagawa@reha.med.keio.ac.jp

T. Kagawa $\cdot$ H. Fukuda

Department of Information and Computer Sciences,

Toyohashi University of Technology, 1-1 Hibarigaoka,

Tempaku-cho, Toyohashi-shi 441-8580, Japan

Y. Uno

Department of Mechanical Science and Engineering,

Graduate School of Engineering, Nagoya University,

Furo-cho, Chikusa-ku, Nagoya-shi 464-8603, Japan 